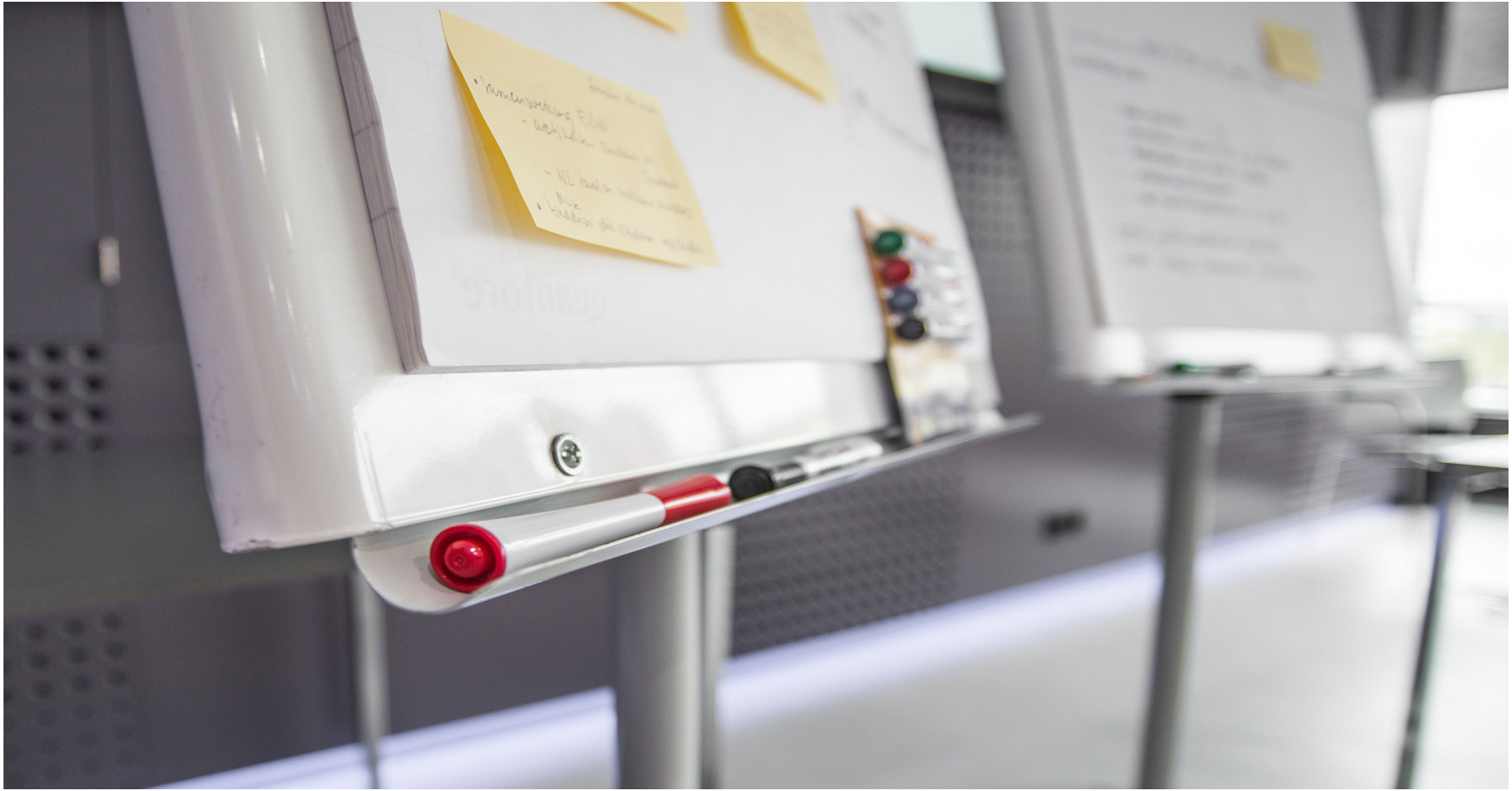

\title{
Análisis Comparativo del Programa de Tecnología en Electrónica Telecomunicaciones en Colombia
}

\section{Comparative Analysis $O F$ the Technology Program in Electronics and Telecommunications in Colombia}

Fabio Pérez-Márquez

Magister en Administración de Datacenters, Fabio.perez@tecnar.edu.co,

Orcid: https://orcid.org/0000-0003-2573-7589, Docente Investigador, Fundación Tecnológica Antonio de Arevalo Unitecnar, Cartagena de Indias, Colombia. 


\section{Resumen}

El presente trabajo, tuvo por objetivo realizar un análisis comparativo de las carreras de Tecnología en Electrónica y Telecomunicaciones en Cartagena, la Costa Atlántica y Colombia. El trabajo fue apoyado por el Centro de Investigaciones Científicas y Tecnológicas - CICTAR de la Fundación Tecnológica Antonio de Arévalo- TECNAR y su grupo de investigación Desarrollo Sostenible Urbano. El alcance del estudio comprendió la revisión de la oferta educativa de ese nivel con base en la información reportada en la plataforma del Sistema Nacional de Información de la Educación Superior - SNIES del Ministerio de Educación Nacional en Colombia - MEN al año 2016. En concreto, el trabajo buscó mostrar el estado actual de la formación en el campo de la profesión para lo cual comparó múltiples variables para los programas existentes por regiones, visualizando las tendencias generales. Para conseguir el objetivo, se utilizaron los métodos y técnicas estadísticas de análisis descriptivo, modelos lineales generalizados y análisis de componentes principales. Entre los resultados, se muestra que los programas así denominados, están dentro de los parámetros académicos nacionales e internacionales y que su denominación es coherente con la naturaleza del campo de conocimiento al cual pertenecen con su respectivo nivel académico, además del grupo de características que los identifican y constituyen particularidades. También se muestra la necesidad y pertinencia del programa en la ciudad de Cartagena y resalta la existencia de programas similares en mayor cuantía en ciertas regiones del país, principalmente en la zona andina, lo cual justifica la necesidad de formación en la disciplina del nivel del programa analizado.

Palabras clave: Currículo, Competencia Ciclo propedéutico,

\section{Abstract}

The objective of this paper was to make a comparative analysis of the Electronics and Telecommunications Technology careers in Cartagena, the Atlantic Coast and Colombia. The work was supported by the Center for Scientific and Technological Research - CICTAR of the Antonio de Arevalo Technology Foundation - TECNAR and its research group Sustainable Urban Development. The scope of the study included a review of the educational offer at this level based on the information reported on the platform of the National System of Information on Higher Education - SNIES of the Ministry of National Education in Colombia - MEN as of 2016. Specifically, the work sought to show the current state of training in the field of the profession by comparing multiple variables for existing programs by region, visualizing general trends. To achieve this objective, the methods and statistical techniques of descriptive analysis, generalized linear models and principal component analysis were used. Among the results, it is shown that the programs so denominated, are within the national and international academic parameters and that their denomination is coherent with the nature of the field of knowledge to which they belong with their respective academic level, besides the group of characteristics that identify them and constitute particularities. It also shows the need and relevance of the program in the city of Cartagena and highlights the existence of similar programs in greater numbers in certain regions of the country, mainly in the Andean area, which justifies the need for training in the discipline of the level of the program analyzed.

Keywords: Curriculum, Competence, Propaedeutic Cycle, Alliance 
Análisis Comparativo de los Programas de Pregrado en Tecnología en Electrónica y Telecomunicaciones en Colombia.

\section{4}

\section{Introducción}

En el contexto mundial se están presentando grandes transformaciones producto de la revolución científica y el uso intensivo de las nuevas tecnologías de la información y comunicación que afectan directamente a la sociedad colombiana y por ende, al sistema educativo. Estos grandes cambios plantean retos a la educación superior exigiendo ofrecer programas pertinentes y con calidad que permitan contribuir al desarrollo de la región y el país. No en vano se reconoce que la educación mantiene un importante rol estratégico y prioritario en la formación y desarrollo humano de profesionales idóneos que se constituyen como parte del capital intelectual trascendental para el desarrollo social y económico, por lo que exige especial atención [1-2].

Autores como [3-4] ratifican la necesidad y conveniencia de implementar y usar estas herramientas y servicios de comunicación e información en las dimensiones didácticas para una real inclusión en la Sociedad del Conocimiento, por cuanto permite la obtención de conocimientos óptimos para afrontar los derroteros anteriormente planteados y es considerada por los estudiantes como una estrategia relevante para su proceso de aprendizaje por cuanto propician ambientes pedagógicos más acogedores y motivadores que permiten al docente presentar los contenidos de una manera más dinámica e interactiva que la empleada en la instrucción tradicional [5-6].

Por ello los gobiernos y sectores educativos y empresariales, buscan trabajar mancomunadamente con el fin de lograr construir de forma conjunta políticas y estrategias encaminadas al fortalecimiento de la formación de profesionales en las nuevas tendencias disciplinares que respondan con pertinencia a las necesidades sectoriales [7]. En este contexto para que las Instituciones
Universitarias puedan lograrlo, es importante incorporar en sus políticas de nivel directivo, la inversión en desarrollo tecnológico y equipamiento acordes con el crecimiento de las mismas, ya que de esta forma sus niveles tecnológicos, por cuanto se constituyen en un valor agregado capaz de crear procesos de calidad y acreditaciones, cobertura, ampliación del portafolio de servicios educativos, la virtualización de sus programas, entre otros; así como brindar condiciones a la sociedad y a su entorno que disminuyan la brecha y el rezago tecnológico presentes [8].

Al respecto de la eficacia, uno de los problemas detectados ha sido la tendencia progresiva de continuar formando profesionales universitarios en carreras tradicionales, cuando los sectores productivos insistentemente plantean la necesidad de formar individuos con competencias y destrezas en hacer y saber hacer. La actual tasa de desempleo en el país y el desempeño laboral en actividades ajenas a los campo de formación del nivel universitario, son una muestra evidente de la saturación del mercado en el ámbito de algunas profesiones por lo que se hace imperativo identificar las reales necesidades de formación que requieren el país y sus regiones con el fin de alcanzar los objetivos de crecimiento proyectados [7].

Así las cosas, La educación superior en Colombia enfrenta retos importantes dentro de los que sobresalen el aumento de niveles de cobertura y el mejoramiento de la calidad de las instituciones que ofrecen los servicios en ese nivel de enseñanza. Si bien, durante las dos últimas décadas el número de estudiantes matriculados ha crecido de manera importante, especialmente en la formación técnica y tecnológica, en un contexto internacional las tasas de cobertura continúan siendo bajas y no superan el $50 \%$. Por otro lado, la calidad del sistema de educación superior es heterogénea, ya que coexisten instituciones 
bien organizadas y reconocidas por su excelencia, con instituciones caracterizadas por bajos niveles de calidad [9], en las cuales juega un papel muy importante en el mejoramiento de la calidad de la educación, la labor docente que desarrollan en el quehacer pedagógico, por cuanto se hace imperante el el reconocimiento de sus esfuerzos que motive un mejor desempeño [10-11].

\section{Marco legal}

Al respecto del marco legal de la educación superior para carreras técnicas y tecnológicas en Colombia, debe decirse que la Constitución Política de 1991 establece que la educación es un derecho fundamental, la cual tiene carácter obligatorio en los nueve primeros años escolares siendo seguida por la educación media la cual es antesala de la educación superior, conocida como el nivel superior de la estructura educativa nacional. Por su parte, es el Ministerio de Educación Nacional (MEN) la entidad encargada de articular el sistema de educación a partir de políticas que comprenden desde los niveles básicos de preescolar hasta llegar a la educación profesional de alto nivel, la cual es competencia del Viceministerio de Educación Superior que mediante las funciones de fomento, inspección y vigilancia, orienta la educación superior con el fin de garantizar el ingreso y la permanencia de los estudiantes en este nivel. Cabe decir que la educación superior colombiana abarca la técnica, la ciencia y la tecnología, las humanidades, el arte y la filosofía en dos niveles, pregrado y postgrado y a su vez, el pregrado ofrece tres niveles de formación, técnica profesional, tecnológica y profesional universitaria, y el postgrado, especialización, maestría y doctorado [1].

Así mismo y refiriéndose al mismo nivel de carreras técnicas y tecnológicas, se informa que el marco legal está constituido básicamente por la Ley 30 de 1992 que organiza el servicio público de la educación superior estableciendo que son aceptadas como establecimientos de la educación superior las Instituciones Técnicas Profesionales, las Instituciones Universitarias o Escuelas Tecnológicas y las Universidades. Esa misma ley concibe la educación "como un proceso permanente que posibilita el desarrollo de las potencialidades del ser humano de una manera integral y tiene por objeto el desarrollo pleno de los alumnos y su formación académica o profesional" y que debe "Profundizar en la formación integral, trabajar por la creación, el desarrollo y la transmisión del conocimiento; promover la utilización del conocimiento para solucionar las necesidades del país y prestar a la comunidad un servicio con calidad. Además ser factor de desarrollo científico, cultural, económico, político y ético".

Por su parte la Ley 749 de 2002 que también regula los niveles anteriormente referenciados y "por la cual se organiza el servicio público de la educación superior en las modalidades de formación técnica profesional y tecnológica”, contiene los lineamientos por los cuales se rigen tanto los programas en las modalidades técnicas profesionales y tecnológicas y las respectivas instituciones de educación superior que se caracterizan por su vocación e identidad manifiesta en los campos de los conocimientos y el trabajo en actividades de carácter técnico, debidamente fundamentadas en la naturaleza de un saber, cuya formación debe garantizar la interacción de lo intelectual con lo instrumental, lo operacional y el saber técnico.

En la misma Ley 749, se encuentran definidos claramente los ciclos de formación, expresando claramente que las instituciones técnicas profesionales y tecnológicas de educación superior organizarán su actividad formativa de pregrado en ciclos propedéuticos así: 
Análisis Comparativo de los Programas de Pregrado en Tecnología en Electrónica y Telecomunicaciones en Colombia.

\section{6}

a) El primer ciclo, estará orientado a generar competencias y desarrollo intelectual como el de aptitudes, habilidades y destrezas al impartir conocimientos técnicos necesarios para el desempeño laboral en una actividad, en áreas específicas de los sectores productivos y de servicios, que conducirá al título de Técnico Profesional.

La formación técnica profesional comprende tareas relacionadas con actividades técnicas que pueden realizarse autónomamente, habilitando para comportar responsabilidades de programación y coordinación.

b) El segundo ciclo, ofrecerá una formación básica común, que se fundamente y apropie de los conocimientos científicos y la comprensión teórica para la formación de un pensamiento innovador e inteligente, con capacidad de diseñar, construir, ejecutar, controlar, transformar y operar los medios y procesos que han de favorecer la acción del hombre en la solución de problemas que demandan los sectores productivos y de servicios del país. La formación tecnológica comprende el desarrollo de responsabilidades de concepción, dirección y gestión de conformidad con la especificidad del programa, y conducirá al título de Tecnólogo en el área respectiva.

c) El tercer ciclo, complementará el segundo ciclo, en la respectiva área del conocimiento, de forma coherente, con la fundamentación teórica y la propuesta metodológica de la profesión, y debe hacer explícitos los principios y propósitos que la orientan desde una perspectiva integral, considerando, entre otros aspectos, las características y competencias que se espera posea el futuro profesional. Este ciclo permite el ejercicio autónomo de actividades profesionales de alto nivel, e implica el dominio de conocimientos científicos y técnicos y conducirá al título de Profesional.
Finalmente se debe informar que el sistema tiene sus reguladores, evaluadores, asesores y prestadores de servicio público. Como reguladores pueden considerarse el Congreso de la República, el Ministerio de Educación Nacional y el Departamento Nacional de Planeación. Estas entidades cumplen funciones de evaluación, además de otros organismos como el Consejo Nacional de Educación Superior - CESU, Consejo Nacional de Acreditación - CNA, Consejo Nacional de Aseguramiento de la Calidad de la Educación Superior - CONACES y los pares académicos. Por su parte, los prestadores del servicio público son las entidades de educación superior que pueden ser públicas y privadas [11-14].

Situación actual de la educación técnica y tecnológica en Colombia

La distribución de la matrícula en Colombia con respecto a la registrada en algunos países de Latinoamérica, presenta grandes diferencias. Según cifras de la UNESCO, en Chile y Uruguay la matrícula en programas técnicos y tecnológicos supera a la del nivel universitario. Si se compara esta proporción con países de Europa como Francia, Reino Unido y Países Bajos, la diferencia se amplía significativamente a favor de la formación técnica profesional y tecnológica [1].

La respuesta sobre cuál distribución es la más favorable para el desarrollo económico y social de un país debe coincidir con los requerimientos del sector productivo y de la brecha que exista en la distribución del ingreso de sus habitantes. En Colombia se evidencia la necesidad de contar con un mayor número de técnicos profesionales y tecnólogos que se especialicen en los sectores que demanda el país para incrementar su productividad y mejorar su competitividad, y al mismo tiempo, que este mayor volumen de población incorporada al mercado laboral genere 
ingresos que contribuyan a disminuir la pobreza y a cerrar la brecha en la distribución del ingreso [15-18].

En ese orden de ideas, el Ministerio de Educación Nacional - MEN, ha venido planteando que para "Aceptar el desafío de contribuir a impulsar el fortalecimiento de la educación técnica y tecnológica y con ello la competitividad y la productividad del país, se requiere conocer el contexto en el que se formulan e implementan las políticas nacionales en el sector educativo "[1],[19].

El mismo MEN expresa que para la oferta de estos programas, el país contó en el año 2008 con 276 instituciones de educación superior que, de acuerdo con la Ley 30 de 1992, se clasificaron, según su carácter, en cuatro grupos: instituciones técnicas profesionales, institutos tecnológicos, instituciones universitarias y universidades. De estas 276 instituciones, 195 eran privadas (71\%) y 81 públicas (29\%); el 33\% del total eran instituciones universitarias, el $26.8 \%$ universidades, el $21.7 \%$ institutos tecnológicos y el restante $18.5 \%$, instituciones técnicas profesionales [1].

En el mismo año, la oferta de la educación superior en Colombia abarcó 55 núcleos básicos del conocimiento en los que se agruparon 5.581 programas de pregrado (706 técnicos profesionales, 1.407 tecnológicos y 3.468 universitarios). Sin embargo, la demanda se concentró en 20 núcleos y de éstos es evidente una preferencia de los estudiantes por carreras tradicionales como Derecho, Administración de Empresas, Contaduría Pública y Medicina.

Históricamente ha existido en el país una preferencia de los estudiantes por los programas universitarios. Un seguimiento a la matrícula en educación superior en los últimos tres años permitió observar una concentración de la matrícula en programas universitarios (74.6\%) frente a programas técnicos profesionales y tecnológicos $(20,7 \%)$. Para contrarrestar los efectos, el Plan Sectorial de Educación 2006-2010 propuso la meta de crear 320.000 cupos en educación superior, de los cuales 200.000 correspondieron a formación técnica y tecnológica, de tal manera que la participación de este nivel en el total de la matrícula de educación superior pasó de 25,7\% en 2006 a 34\% en 2010 [1].

\section{Materiales y métodos}

El alcance del estudio comprendió la revisión de la oferta educativa del nivel tecnológico en el área de Electrónica y Telecomunicaciones en Cartagena, Costa Atlántica y Colombia con base en la información reportada en la plataforma del Sistema Nacional de Información de la Educación Superior - SNIES del Ministerio de Educación Nacional en Colombia - MEN al año 2016.



Grafica 1: Colombia - Cartagena

A continuación se describen las etapas del procesamiento de datos que permitió realizar el análisis comparativo de la carrera de nivel tecnológico en estudio. 


\section{8}

\section{Procesamiento de la información}

a) La información correspondiente a las carreras relacionadas con Tecnología en Electrónica y Telecomunicaciones se recopiló del SNIES, sistema que recopila y organiza la información relevante sobre la educación superior que permite hacer planeación, monitoreo, evaluación, asesoría, inspección y vigilancia del sector. Este sistema como fuente de información, en relación con las instituciones y programas académicos aprobados por el Ministerio de Educación Nacional, consolida y suministra datos, estadísticas e indicadores considerados como relevantes, confiables y oportunos. De igual forma se contrastó aleatoriamente la existencia de las carreras en las páginas web de las instituciones que los reportan.

b) A continuación se procedió a realizar las graficación individual con las respectivas valoraciones obtenidas al año 2016. Se ubicaron los programas activos e inactivos, distribución por sectores; metodologías, ciclos propedéuticos, cantidad de créditos, carácter de instituciones ofertantes, distribución por departamentos y costo entre otras variables.

c) Finalmente se organizó la información y se procedió con el análisis comparativo por cada gráfica generada por los modelos institucionales en el periodo anteriormente referenciado.

Resultados

Análisis de carreras tecnológicas relacionados con el área de Electrónica.

- Distribución nacional del programa (TET) y afines.

A nivel de distribución nacional, se encontró que de los 131 programas afines reportados en el SNIES, 21 se encuentran activos y 110 inactivos.

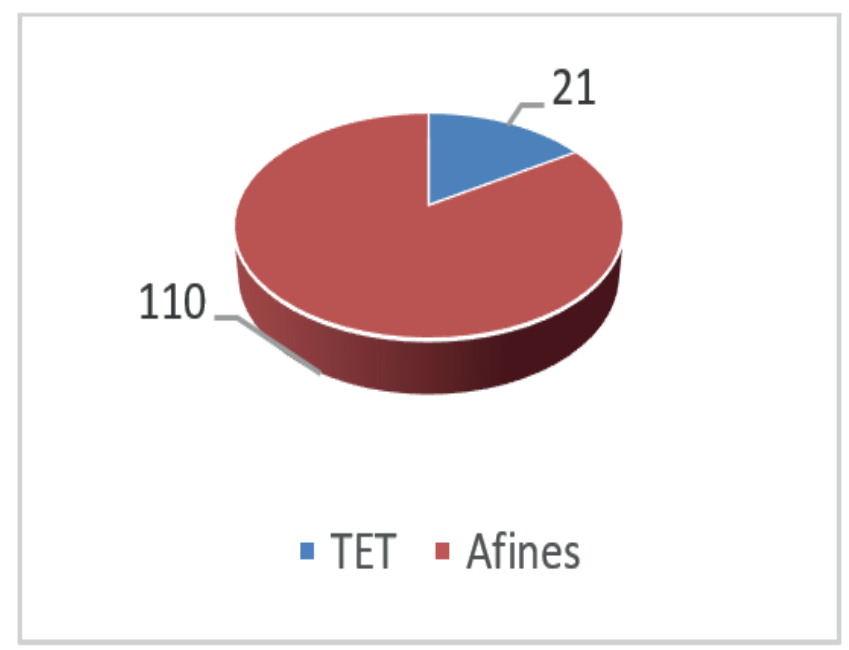

Grafica 2: Distribución Nacional del programas. Col. 2016.

-Programa(TET) frenteaotras denominaciones del área.

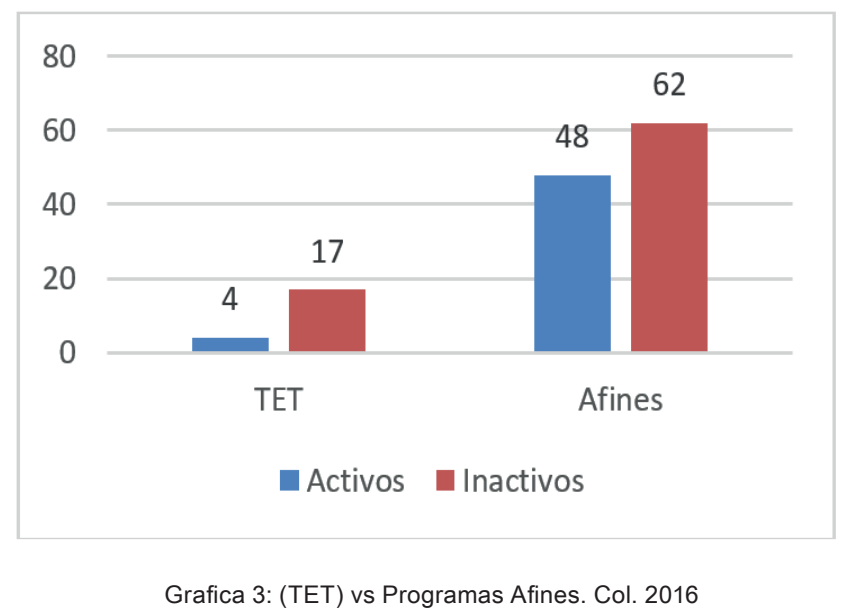

Se encontró que del total de los 131 programas relacionados con el área de estudio a nivel nacional, solo 21 corresponden a denominaciones específicas del programa de Tecnología en Electrónica y y Telecomunicaciones de los cuales únicamente cuatro (4) se encuentran activos. Adicionalmente se pudo observar que los 110 restantes corresponden a denominaciones similares y afines, encontrándose activos únicamente 48 programas. 
- Distribución Nacional (TET) x Regiones

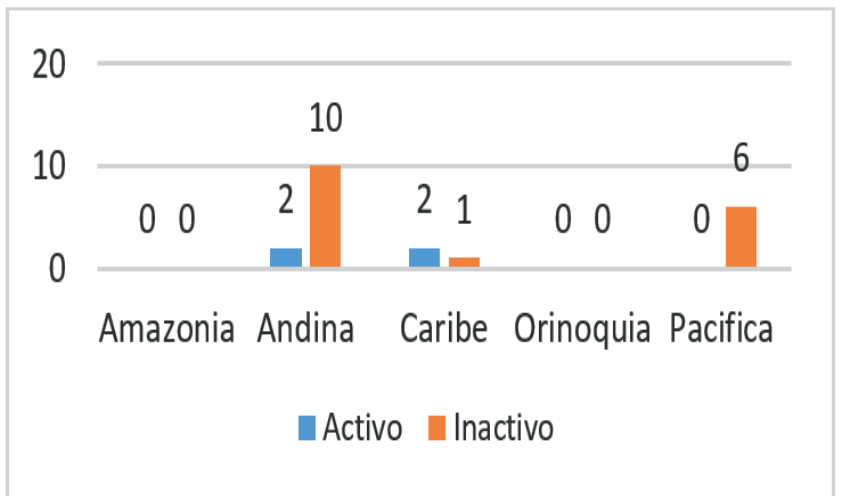

Grafica 4: (TET) Distribución x Regiones. Colombia. 2016

En cuanto a la distribución nacional del programa de (TET) en las cinco (5) regiones del país, se encontró que únicamente dos (2) programas activos hacen presencia en la región caribe colombiana y 2 activos en la región Amazonía, Orinoquía y Pacifica no reportan existencia del programa activo.

- Modalidad empleada por el programa (TET) a nivel nacional.

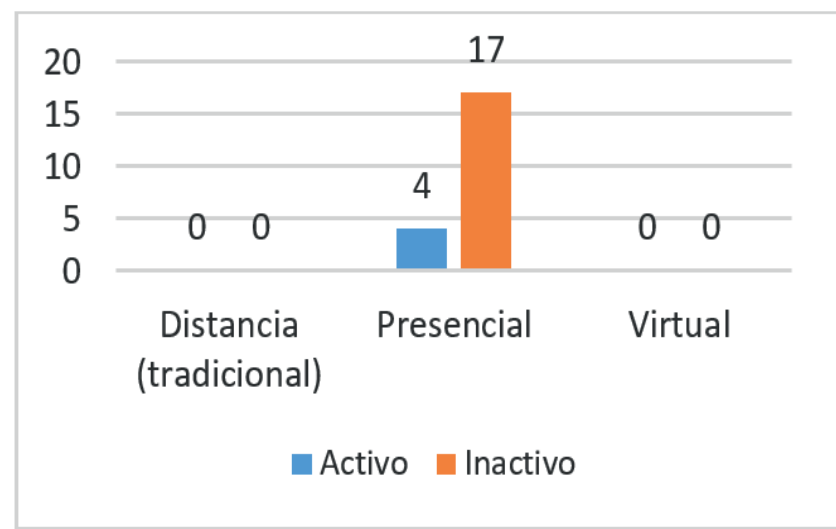

Grafica 5: (TET) Modalidad. Colombia. 2016

De acuerdo a la información reportada en SNIES, se pudo observar que de los 21 programas de (TET) a nivel nacional corresponden a la modalidad presencial; con cuatro (4) programas activos y 17 Inactivos. No reportan en Distancia y Virtual.
- Sectorización Programa (TET) en Colombia.

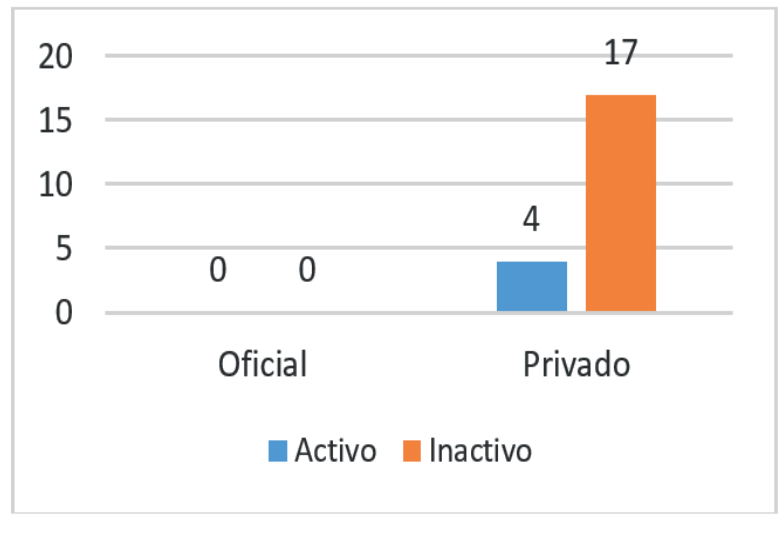

Grafica 6: (TET) Sectorización por regiones. Col. 2016

La sectorización relacionada con un programa académico de cualquier nivel, está relacionada con el tipo de institución educativa que lo ofrece. Así las cosas, se encontró que el programa (TET) a nivel nacional solamente es ofertado por instituciones privadas

- (TET) por ciclos propedéuticos en Colombia

Los ciclos propedéuticos ofrecen al estudiante la opción de cursar una carrera de forma fragmentada, lo cual permite que éste pueda insertarse en el campo laboral con la posibilidad de continuar a futuro sus estudios en un nivel superior.

Para el programa de (TET), se pudo observar que en la actualidad esta Tecnología no se oferta por ciclos propedéuticos

- (TET) Número de créditos en Colombia. 2016

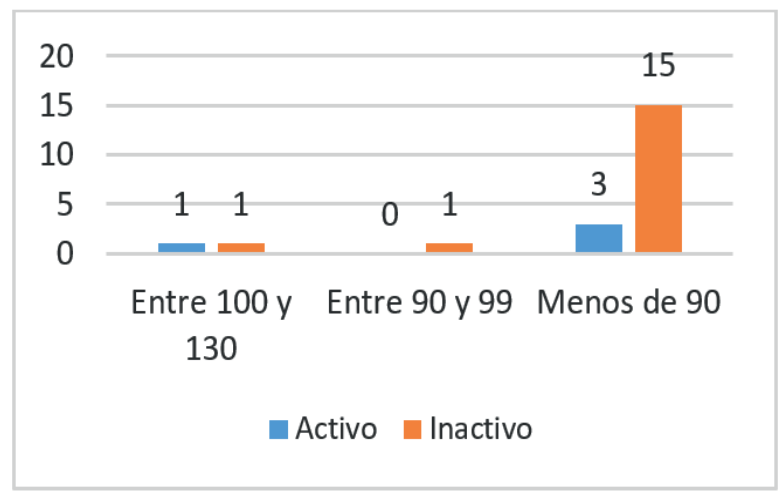

Grafica 7: (TET) Numero de créditos. Col. 2016 
Análisis Comparativo de los Programas de Pregrado en Tecnología en Electrónica y Telecomunicaciones en Colombia.

\section{0}

El crédito es la unidad de medida que representa el tiempo de dedicación del estudiante para el desarrollo de la actividad académica. En concreto, hace referencia a la dedicación con apoyo del docente y al tiempo de trabajo independiente.

Para el programa en cuestión, se pudo observar que de los cuatro (4) programas activos, tres (3) Reportan número de créditos menores a 90 y solo uno (1) se reporta con más de 100 créditos

\section{Conclusiones y Discusiones}

Se encontró que del total de los 131 programas relacionados con el área de estudio a nivel nacional, solo 21 corresponden a denominaciones específicas del programa de Tecnología en Electrónica y Telecomunicaciones de los cuales únicamente cuatro (4) se encuentran activos. Adicionalmente se pudo observar que los 110 restantes corresponden a denominaciones similares y afines, encontrándose activos únicamente 48 programas. En cuanto a la distribución nacional del programa de (TET) en las cinco (5) regiones del país, se encontró que únicamente dos (2) programas activos hacen presencia en la región caribe colombiana y 2 activos en la región Amazonía, Orinoquía y Pacifica no reportan existencia del programa activo. En el caribe son ofertados por la Fundación Tecnológica Antonio de Arévalo en Cartagena y en Barranquilla. La existencia de solo nueve (4) programas activos de Tecnología en Electrónica y Telecomunicaciones en Colombia, dan cuenta de que el programa podría responder a una demanda cautiva o nicho existente relacionado con la disciplina y necesidades de sectores empresariales debido a la escasa oferta educativa. En resumen el análisis realizado al estado actual del programa de Tecnología en Electrónica y Telecomunicaciones y afines ofertado por la Fundación Tecnológica Antonio de Arévalo - TECNAR bajo la denominación de Tecnología en Salud ocupacional, permitió valorar hasta qué punto el programa presenta semejanzas con otros a nivel nacional, lo cual muestra la universalidad del mismo. De igual forma, quedó demostrado que el programa se inscribe dentro de las tendencias del campo al cual pertenece. El estudio también permitió comparar el estado de la formación del programa en cuanto a la tendencia nacional de las modalidades en las que se ofrecen programas similares en Colombia.

\section{Referencias}

[1] Educación técnica y tecnológica para la competitividad. 2008. [En línea]. Disponible en:www.mineducacion.gov.co/1621/ articles176787_archivo_pdf.pdf.

[2] L. Jiménez Rodríguez y R. Gamboa-Suárez, “El capital humano e intelectual como catalizador de la gestión organizacional", Mundo Fesc, vol. 8, no. 15 , pp. 83-89, 2018.

[3] M.M. Martín, C.A. Hernández-Suarez, y S.M. Mendoza-Lizcano, "Ambientes de aprendizaje basados en herramientas web para el desarrollo de competencias TIC en la docencia", Perspectivas, vol. 2, n. ${ }^{\circ}$ 1, pp. 97-104, 2017

[4] C.L. Gómez Valderrama y R.H. Ramírez Sánchez, "Comunidades de aprendizaje móvil, Mastery Learning y el problema 2 sigma como estrategias para el conocimiento previo, en la resolución de actividades", Ecomatematico, vol. 8, pp. 25-32, ene. 2017

[5] A.M. Rogriguez-Suarez, J.A. MorenoMontagut, y M. Trigos-Rodriguez, "Los videos tutoriales como herramienta formativa", Revista Ingenio, vol. 10, no. 1, pp. 37-42, 2016

[6] M.C. Ospina-Hérnandez, A.M. SuarezCastrillon, G. Espinoza-Morales, y N. JaimesSandoval, "Uso de las TIC despierta una mayor motivación que con la no inclusión 
de las mismas en el proceso de enseñanza y aprendizaje", Revista Ingenio, vol. 9, no, 1, pp. 101-119, 2016

[7] Panorama de las Carreras Técnicas y Tecnológicas en Colombia. 2005. [En línea]. Disponble en: http://www.laccei.org/ LACCEI2005Cartagena/Papers/ED132_ MartinezPalmera.pdf

[8] M.M. Peñaranda-Peñaranda, R.A. BayonaTrigos, y O. Manzano-Durán, "Las tecnologías de la información y la comunicación: una herramienta estratégica para el desarrollo académico y profesoral", Revista Ingenio, vol. 14, n. ${ }^{\circ}$ 1, pp. 93-102, 2017

[9] L. Melo, J. Ramos, P. Hernández, “La educación superior en Colombia: situación actual y análisis de eficiencia”, Revista Desarrollo y Sociedad, 2005.

[10] J.A. Parada Puentes, "Estrategias Gerenciales para el Reconocimiento del Desempeño Laboral Docente", Mundo Fesc, vol. 7, no. 14, pp. 42-56, 2017

[11] J. Hernández-Sánchez, E. Borjón-Robles, y M. Torres-Ibarra, "Dimensiones de la tecnología en la formación inicial de profesores de matemáticas: un estudio desde el currículum oficial”, Ecomatemático, vol. 7, no. 1, pp. 6-19, ene. 2016

[12] Departamento Nacional de Planeación. [En línea]. Disponible en: http://www.dnp.gov.co/

[13] Plan Nacional de Desarrollo. Estado Comunitario: desarrollo para todos. [En línea]. Disponible en: http://www.dnp.gov.co/ paginas_detalle. aspx?idp=699

[14] Visión Colombia II Centenario: 2019. [En línea]. Disponible en: http://www.dnp.gov.co/ paginas_detalle. aspx?idp $=366$
[15] Alta Consejería Presidencial para la Competitividad y Productividad. [En línea]. Disponible en: http://www.snc.gov.co/pagina_ nueva/consejero/funciones.html

[16] Comisión Nacional de Competitividad. [En línea]. Disponible en: http://www.snc.gov.co/ pagina_nueva/nacional. html

[17] Comisiones Regionales de Competitividad. [En línea]. Disponible en: http://www.snc.gov. co/pagina_nueva/regionales. html

[18] Sistema Nacional de Competitividad. [En línea]. Disponible en: http://www.snc.gov.co/ pagina_nueva/index.html

[19] Bases de la política para el diseño de programas de educación superior por ciclos y competencias. Ministerio de Educación Nacional. [En línea]. Disponible en: http://www.mineducacion.gov. co/1621/article- 131953.html 